\title{
Sulfur chemistry: 1D modeling in massive dense cores
}

\author{
V. Wakelam ${ }^{1,2}$, F. Hersant ${ }^{1,2}$, and F. Herpin ${ }^{1,2}$ \\ 1 Université de Bordeaux, Observatoire Aquitain des Sciences de l’Univers, 2 rue de l'Observatoire, BP 89, 33271 Floirac Cedex, \\ France \\ 2 CNRS, UMR 5804, Laboratoire d'Astrophysique de Bordeaux, 2 rue de l'Observatoire, BP 89, 33271 Floirac Cedex, France \\ e-mail: wakelam@obs.u-bordeaux1.fr
}

Received 18 November 2010 / Accepted 18 February 2011

\begin{abstract}
Aims. The main sulfur-bearing molecules OCS, $\mathrm{H}_{2} \mathrm{~S}, \mathrm{SO}, \mathrm{SO}_{2}$, and CS have been observed in four high mass dense cores (W43-MM1, IRAS 18264, IRAS 05358, and IRAS 18162). Our goal is to put some constraints on the relative evolutionary stage of these sources by comparing these observations with time-dependent chemical modeling.

Methods. We used the chemical model Nahoon, which computes the gas-phase chemistry and gas-grain interactions of depletion and evaporation. Mixing of the different chemical compositions shells in a 1D structure through protostellar envelope has been included since observed lines suggest nonthermal supersonic broadening. Observed radial profiles of the temperature and density are used to compute the chemistry as a function of time.

Results. With our model, we underproduce CS by several orders of magnitude compared to the other S-bearing molecules, which seems to contradict observations, although some uncertainties in the CS abundance observed at high temperature remain. The $\mathrm{OCS} / \mathrm{SO}_{2}, \mathrm{SO} / \mathrm{SO}_{2}$, and $\mathrm{H}_{2} \mathrm{~S} / \mathrm{SO}_{2}$ abundance ratios could in theory be used to trace the age of these massive protostars since they show a strong dependence with time, but the sources are too close in age compared to the accuracy of chemical models and observations. Our comparison between observations and modeling may, however, indicate that W43-MM1 could be chemically younger than the three other sources. Turbulent diffusivity through the protostellar envelopes has to be less efficient than $2 \times 10^{14} \mathrm{~cm}^{2} \mathrm{~s}^{-1}$. Otherwise, it would have smoothed out the abundance profiles, and this would have been observed.

Conclusions. The sulfur chemistry depends strongly on the 1D physical conditions. Any observed set of abundances should be compared with a chemical model computed with the same temperature and density traced by the observations. In our case, no conclusion can be given on the relative age of IRAS 18264, IRAS 18162 and IRAS 05358 except that they are very close. W43-MM1 seems younger than the other sources. Turbulent mixing could occur in young high-mass protostars on a geometric scale that is too small to affect the chemical abundance profiles through the envelope.
\end{abstract}

Key words. astrochemistry - turbulence - stars: formation - ISM: molecules

\section{Introduction}

Despite our poor knowledge of the sulfur chemistry in the interstellar medium, the abundance of S-bearing molecules observed in protostars has been used to put some constraints on the age of the sources being studied (Charnley 1997; Hatchell et al. 1998; Buckle \& Fuller 2003; van der Tak et al. 2003; Wakelam et al. 2004; Herpin et al. 2009). Most of the rate coefficients of the reactions involving sulfur-bearing molecules have not been studied in the laboratory because of the difficulty of conducting experiments with them. Rate coefficients are then uncertain and many must be taken with caution. Another problem related to sulfur chemistry is the depletion of the element. Observations of the atoms in the diffuse medium have shown a relationship between the amount of depletion of the elements (in a more or less refractory form) with the density of the cloud (see Jenkins 2009, and references therein). Sulfur is one of the exceptions that do not show this depletion. As far as observations can go, the atomic abundance seems to be the same as the cosmic sulfur abundance of about $1.5 \times 10^{-5}$ compared to the proton density (Sofia \& Meyer 2001). If using such an elemental abundance in chemical models for cold clouds, we would overpredict gas phase abundances of $\mathrm{SO}, \mathrm{SO}_{2}$, and CS by several orders of magnitude (Ruffle et al. 1999). For this reason, a depletion of this element is usually assumed to proceed in dense clouds where the amount of $\mathrm{S}$ available for the chemistry is taken to be two orders of magnitude less than the cosmic abundance. Recently, by reanalyzing hundreds of observations of atomic sulfur line, Jenkins (2009) has argued that not observing the sulfur depletion in diffuse medium is an observational bias. If confirmed, this result would solve one of the interstellar sulfur mysteries.

The form of sulfur on interstellar grains is not known. OCS is the only molecule actually observed on interstellar ices (Palumbo et al. 1997). Since hydrogenation is expected to be the most efficient process on grains, $\mathrm{H}_{2} \mathrm{~S}$ is also probably formed, although with a smaller abundance than the observational limit of $10^{-7}$ (van Dishoeck \& Blake 1998). $\mathrm{H}_{2} \mathrm{SO}_{4}$ has been proposed and looked for without any success (Scappini et al. 2003). Finally, Wakelam et al. (2004) propose that sulfur is in the form of polymers or aggregates of sulfur that would be quickly converted into atomic sulfur once evaporated in the gas phase. In protostellar envelopes, when the temperature increases, the sulfured molecules evaporated from the grains are transformed into SO first and then into $\mathrm{SO}_{2}$ (Charnley 1997; Wakelam et al. 2004). Thus abundances of these species have been used on several occasions to trace the chemical evolution of such objects.

Van der Tak et al. (2003) have studied the sulfur chemistry in nine high-mass protostars and find that high-energy transitions probing the inner parts of the protostars where the temperature exceeds $100 \mathrm{~K}$ were mandatory for using S-bearing molecules as 
chemical clocks. They were only able to obtain the abundance of $\mathrm{SO}_{2}$ in these regions. In addition, they propose that OCS is the main carrier of sulfur on grains, based on the high excitation temperature of the molecule and its high abundance in the protostars.

In the present work, we made a $1 \mathrm{D}$ modeling of the sulfur chemistry in four high-mass compact objects, IRAS 18264, IRAS 18162, IRAS 05358, and W43-MM1, presumably representative of the youngest stages of massive star formation. We used the density and temperature profiles determined from a detailed modeling of the SED observed in these sources by Herpin et al. (2009). Herpin et al. (2009) also observed the main $\mathrm{S}$-bearing molecules in these sources in order to get an estimation of their abundance and possible evolutionary stage. The observed line profiles have shown a non-thermal line broadening that they attribute to supersonic diffusion. We then included a treatment of the diffusion in our chemical modeling in order to study its impact on the abundance profiles computed by the model.

This paper is organized as follows. Our 1D chemical model and the model parameters are described in Sect. 2. Section 3 shows the abundance (compared to $\mathrm{H}$ ) and abundance ratio profiles computed by the model in the studied sources, as well as the effect of turbulent mixing. We discuss the particular problem of CS in Sect. 4. We compare our model predictions with the observed abundances in Sect. 5 and conclude in the last section.

\section{Model description}

The model used for this study is the Nahoon chemical model developed by Wakelam et al. (2005, 2010). This code originally computes the chemical evolution of a list of species as a function of time for a fixed temperature and density for a single point. In this new version of Nahoon, the chemical evolution is computed in a 1D structure and accounts for diffusive mixing induced by turbulent transport. Starting from the chemical composition of a molecular cloud (see Sect. 2.3), we then compute the chemical abundances as a function of the radius to the center of the protostars and as a function of time using the physical structures (density and temperature profiles) observed in the four high-mass protostars described in Sect. 2.4. We compute the evolution of chemical abundances $x_{i}$ following

$$
\frac{\partial x_{i}}{\partial t}=P_{i}-L_{i}+\frac{1}{\rho r^{2}} \frac{\partial}{\partial r} \kappa \rho r^{2} \frac{\partial}{\partial r} x_{i}
$$

where $P_{i}$ and $L_{i}$ are the chemical production and loss terms, $\rho$ is the density, $\kappa$ the turbulent diffusivity, and $r$ the spherical radius. We compute turbulent diffusion in spherical coordinates.

\subsection{The chemistry}

Gas-phase chemistry is computed and species are allowed to deplete on grains and evaporate by direct temperature effect and indirect heating by collisions between grains and cosmic ray particles. No reactions on grains are taken into account in this work. Only an approximation for the formation of $\mathrm{H}_{2}$ on grains is considered following Leung et al. (1984). The freezeout of gasphase species onto grains is computed using Eq. (1) of Hasegawa et al. (1992). The evaporation of species from the grain mantles is also computed according to Hasegawa et al. (1992) for direct thermal evaporation and from Hasegawa \& Herbst (1993) for evaporation induced by collisions between grains and cosmicray particles.

The chemistry is described by the Srates network from Wakelam et al. (2004). Srates is a reduced network dedicated to sulfur chemistry in warm sources. It contains 929 chemical reactions involving 76 gas-phase and 32 surface species for the elements $\mathrm{H}, \mathrm{He}, \mathrm{C}, \mathrm{O}$ and $\mathrm{S}$. This network has been assembled from different sources (OSU, UDFA and the NIST databases) and updated using the osu latest network (osu-09-2008, http://www . physics.ohio-state.edu/ eric/research.html). To select the reduced network, several previous works were followed (Ruffle et al. 2002; Hollenbach \& McKee 1979; Hartquist et al. 1980; Pineau Des Forêts et al. 1993; Charnley 1997) and the results of simulations obtained with this reduced network were tested against the ones of larger networks valid at low temperature. Srates can be downloaded at the following address: http://kida.obs.u-bordeaux 1.fr/models. The binding energies for OCS and $\mathrm{H}_{2} \mathrm{~S}$ are taken to be 2888 and $2743 \mathrm{~K}$, respectively. These binding energies were computed using equation 1 described in Collings et al. (2004) and the evaporation temperatures of these species measured by TPD experiments from the same paper (Herma Cuppen, private communication). $\mathrm{H}_{2}$ cosmic-ray ionization rate is $1.3 \times 10^{-17} \mathrm{~s}^{-1}$.

\subsection{Diffusion}

Nonthermal broadening of lines is observed in various prestellar cores. This behavior is usually assumed to be the consequence of some sort of unresolved gas motions, whose exact origin and properties are unknown. However, we assume that these motions produce some kind of mixing inside the source and are often referred to as turbulence. Our purpose is to investigate the exact influence of a turbulent-like mixing on the distribution of S-bearing species, without presuming the exact nature of this "turbulence". The simplest way to do so is to use a mixing length approach (Prandtl 1925). We added mixing terms to all the differential equations in order to couple each spatial point in the protostar envelopes with its adjacent points. Turbulence induces an enhanced diffusion in the source, whose effective diffusivity can be written as

$\kappa=v_{\mathrm{T}} l_{\mathrm{T}}$

where $v_{\mathrm{T}}$ and $l_{\mathrm{T}}$ are a characteristic turbulent velocity and length scale (the mixing length), respectively. Expressing $v_{\mathrm{T}}$ and $l_{\mathrm{T}}$ would require a detailed model for turbulence in protostellar cores. This is not, however, within the scope of the paper. Instead, we aim to use observed quantities as upper limits for both parameters. To estimate the maximum efficiency of the turbulent diffusion, we assume that the velocity $v_{\mathrm{T}}$ cannot be higher than the sound speed $\left(\sqrt{\frac{R T}{\mu}}\right.$, with $R$ the gas constant, $T$ the gas temperature, and $\mu$ the mean molecular weight), and the mixing length $l_{\mathrm{T}}$ is expected to be smaller than the radius of the envelope $\left(\sim 3 \times 10^{17} \mathrm{~cm}\right)$. We obtain a maximum turbulent diffusivity $\kappa_{\max }$ of about $2 \times 10^{22} \mathrm{~cm}^{2} \mathrm{~s}^{-1}$.

\subsection{Initial conditions for the protostellar envelope}

Protostars form from dense clouds. As a consequence, for the initial conditions of the protostars, we computed the chemical composition of a dense molecular cloud with a temperature of $10 \mathrm{~K}$, a total $\mathrm{H}$ density of $2 \times 10^{4} \mathrm{~cm}^{-3}$, a visual extinction of 30 , and a cosmic-ray ionization rate of $1.3 \times 10^{-17} \mathrm{~s}^{-1}$. For the dense cloud, species are assumed to be initially in the atomic form 
Table 1. Grain mantle composition.

\begin{tabular}{lcc}
\hline \hline Species & Abundance (/H) & Ref. \\
\hline $\mathrm{CO}$ & $4 \times 10^{-6}$ & $(1)$ \\
$\mathrm{H}_{2} \mathrm{O}$ & $5 \times 10^{-5}$ & $(2)$ \\
$\mathrm{H}_{2} \mathrm{CO}$ & $2 \times 10^{-6}$ & $(3)$ \\
$\mathrm{CH}_{4}$ & $5 \times 10^{-7}$ & $(4)$ \\
$\mathrm{CH}_{3} \mathrm{OH}$ & $2 \times 10^{-6}$ & $(5)$ \\
$\mathrm{OCS}$ & $5 \times 10^{-8}$ & $(6)$ \\
$\mathrm{H}_{2} \mathrm{~S}$ & $5 \times 10^{-8}$ & \\
$\mathrm{~S}(\bmod 1)$ & $1.45 \times 10^{-5}$ & \\
$\mathrm{~S}(\bmod 2)$ & 0 & \\
\hline
\end{tabular}

References. (1) Gibb et al. (2000), (2) Schutte et al. (1996), (3) Keane et al. (2001), (4) Chiar et al. (1996), (5) Boogert et al. (1998), (6) Palumbo et al. (1997).

(coming from the diffuse medium) except for $\mathrm{H}_{2}$. Elemental abundances (compared to total $\mathrm{H}$ ) used for this calculation are 0.09 for He, $2.56 \times 10^{-4}$ for $\mathrm{O}$ and $1.2 \times 10^{-4}$ for $\mathrm{C}^{+}$(Wakelam $\&$ Herbst 2008). For $\mathrm{S}^{+}$, we chose an elemental abundance of $5 \times 10^{-7}$ for the dense cloud in order to approximately reproduce the observed abundances of gas-phase S-bearing molecules (see Wakelam et al. 2004). Using our reduced network, we have to integrate over a long time $\left(10^{7} \mathrm{yr}\right)$ to obtain abundances that are similar to the ones observed in dense clouds. We in fact obtain abundances similar to our previous study (Wakelam et al. 2004, Composition A of Table 1). This time is probably too long compared to the age of dense molecular clouds. Using larger networks of more than four thousand gas-phase reactions, observations in dense clouds are usually reproduced at a few $10^{5} \mathrm{yr}$ (see for instance Wakelam et al. 2006). The reason we need more time is most likely that we have fewer chemical species and that it takes more time for the ionization fraction of the gas to decrease. At high temperatures however, we do not have this problem. This calculation gives us our gas-phase composition prior to the formation of the protostar. The $\mathrm{CO}$ abundance computed by the model on the grain surfaces (result of the depletion of gas-phase $\mathrm{CO}$ ) is about ten times larger than what is observed. We decreased this value to $4 \times 10^{-6}$ (CO abundance observed in interstellar ices towards the massive protostar line of sight W33 by Gibb et al. 2000) and put the rest of the CO back in the gas phase. The total CO gas-phase abundance is then $6.08 \times 10^{-5}$. This change will not affect our chemistry since most of the protostar envelopes are above the evaporation temperature of $\mathrm{CO}$ $(\sim 18 \mathrm{~K})$. Like most dense cloud chemical models, our simulations fail to reproduce the observed limits on the $\mathrm{O}_{2}$ abundance in dense clouds, which is $5 \times 10^{-8}$ (compared to $\mathrm{H}$ ) in L134N (Pagani et al. 2003), 160 times smaller than the abundance computed by our model. We then decreased the gas-phase abundance to its observed limit, and the rest of the oxygen we put in the abundance of atomic oxygen. The computed $\mathrm{O}_{2}$ abundance on grains is $5 \times 10^{-5}$. For $\mathrm{H}_{2} \mathrm{O}$, our model predicts a gas-phase abundance of $4 \times 10^{-8}$, below the observational limit in L134N $\left(1.5 \times 10^{-7}\right.$ Snell et al. 2000) so we did not change it.

Since we did not compute the grain surface chemistry occurring during the dense cloud phase, we modified the grain surface composition obtained from our modeling. For the species observed on interstellar grains, we adopted the abundances listed in Table 1 (see Wakelam et al. 2004). Note that $\mathrm{CO}_{2}$, one of the main constituent of grain mantles, is not present in our network. OCS is the only S-bearing species observed in the solid state in the interstellar medium. $\mathrm{H}_{2} \mathrm{~S}$ is highly suspected of being present, although at a nondetectable level. We set its abundance
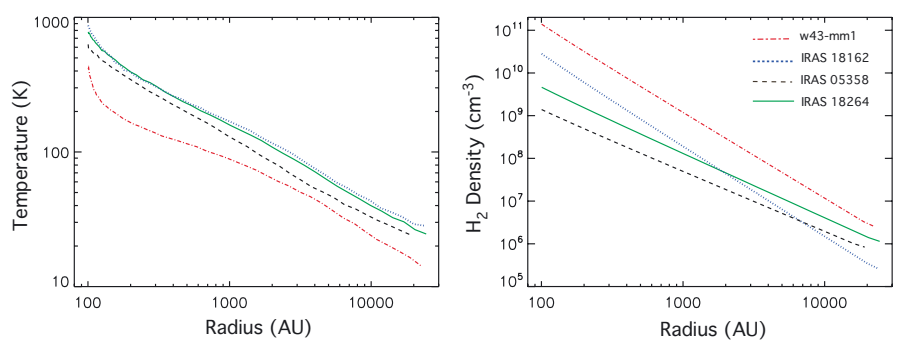

Fig. 1. Temperature and density profiles in the four sources from Herpin et al. (2009).

on grain surfaces to the upper limit calculated by van Dishoeck \& Blake (1998). All the rest of the sulfur was assumed to be either in the atomic form on grains, our model 1, or in a refractory form that cannot evaporate at the temperatures of hot cores, our model 2. This gas-phase and grain mantle composition is used as initial conditions for the whole envelope of the four protostars we studied. Since we do not include any chemical reaction on the grain surfaces, the gradual depletion of the species during the evolution of the chemistry only affects the fraction of species in the gas and on the surface and does not change the chemistry itself.

\subsection{Selected sources: physical structure and observed abundances}

The source sample from Herpin et al. (2009) is made of two IR-quiet dense cores (MSX Flux $21 \mu \mathrm{m}<10 \mathrm{Jy}$ at $1.7 \mathrm{kpc}$, following the definition of Motte et al. 2007) and two slightly brighter ones (IRAS $05358+3543$ and IRAS 18162-2048), with bolometric luminosities $(0.6-2.3) \times 10^{4} L_{\odot}$ at distances $1.8-5.5 \mathrm{kpc}$ and sizes of $\sim 0.11-0.13 \mathrm{pc}$. These massive dense cores are meant to represent the earliest phases of the high-mass star formation.

From the fitted SEDs and the literature, Herpin et al. (2009) propose a rough evolutionary classification of the four objects (W43-MM1 being the youngest object): W43-MM1 $\rightarrow$ IRAS 18264-1152 $\rightarrow$ IRAS 05358+3543 $\rightarrow$ IRAS 18162-2048. Nevertheless and despite this youth, W43MM1 appears to lie apart from the other massive dense cores as it has very likely already developed a hot core.

The temperature and density profiles for the four sources have been derived by Herpin et al. (2009) from the analysis of the spectral energy distribution (SED) with the radiative transfer code MC3D (Wolf et al. 1999) (see also Marseille et al. 2008). The profiles are shown in Fig. 1. Temperatures and densities increase towards the center of the sources. The three IRAS sources show very similar profiles, whereas W43-MM1 is denser and colder. For the chemical modeling, we assumed that the gas temperature and density are abruptly raised from the cold molecular phase to the ones currently observed following previous works (Doty \& Neufeld 1997; van der Tak \& van Dishoeck 2000; Doty et al. 2002). This is of course a strong assumption but we prefer not to add another uncertain parameter. Considering the fast evolution of sulfur chemistry at high temperature (see Sect. 3.1), we do not expect this assumption to affect our main conclusions. Only a careful coupling of the chemistry with a dynamical infalling envelope can, however, answer that question.

Herpin et al. (2009) observed several transitions of OCS, $\mathrm{H}_{2} \mathrm{~S}, \mathrm{SO}, \mathrm{SO}_{2}$, and $\mathrm{CS}$ in the four sources, as well as some of their ${ }^{34} \mathrm{~S}$ minor isotopologues. They constrained the species abundances at different radii depending on their excitation temperatures $(60,75$, and $100 \mathrm{~K})$ (see Table 5 of their paper). We 
Table 2. S-bearing molecular abundances $\left(\times 10^{-10}\right)$ derived in the four sources by Herpin et al. (2009).

\begin{tabular}{lccccc}
\hline \hline Molecule & $T(\mathrm{~K})$ & \multicolumn{4}{c}{ Sources } \\
& & w43-mm1 & IRAS 18264 & IRAS 05358 & IRAS 18162 \\
\hline $\mathrm{CS}$ & 60 & 36 & 4.0 & 2.3 & - \\
& $100^{a}$ & 160 & 1.1 & - & 4.6 \\
$\mathrm{OCS}$ & 60 & 92 & 2.5 & 2.4 & 1.4 \\
& 100 & 130 & 1.1 & 3.7 & 3.3 \\
$\mathrm{H}_{2} \mathrm{~S}$ & 60 & 3.0 & 1.3 & 1.2 & 3.1 \\
& 100 & 4.0 & 0.15 & 0.51 & 0.30 \\
$\mathrm{SO}$ & 60 & 0.90 & 0.48 & 1.1 & 2.4 \\
& 100 & 24 & 0.64 & - & 3.6 \\
$\mathrm{SO}_{2}$ & 60 & 2.0 & 0.42 & 0.62 & 1.7 \\
& 100 & 160 & 2.0 & 6.4 & 7.6 \\
\hline
\end{tabular}

Notes. ${ }^{(a)}$ The CS abundance at this excitation temperature was derived using the $\mathrm{CS}$ (7-6) intensity line blended with an $\mathrm{H}_{2} \mathrm{CO}$ line (see Herpin et al. 2009). The symbol - means that the no abundance was derived because the molecular line has not been observed.

report in Table 2 their observed abundances for excitation temperatures of 60 and $100 \mathrm{~K}$. We assume that we are at LTE so the excitation temperature of the gas reflects the gas and dust temperatures. The presence of a hot core in W43-MM1 might explain the observed higher abundance of $\mathrm{H}_{2} \mathrm{~S}$ and OCS in that source relative to the other ones. Moreover, strong shocks within this source probably influence the chemistry. One surprising result is the small abundance of the S-bearing molecules found in all four sources compared to the cosmic abundance (three to four orders of magnitude smaller). We compare our modeling with their observational results below.

\section{Modeling results}

\subsection{Abundances through the protostellar envelopes}

Figures 2 and 3 show the abundances of the main S-bearing molecules computed by the chemical model as a function of the radius to the central object in the envelope of the four protostars at $10^{4} \mathrm{yr}$, which was approximately the age derived by Doty et al. (2002) for the massive protostar AFGL 2591. This age was derived by comparing the predictions of chemical models with a large number of molecules observed in this source. Although AFGL2591 may be a bit more evolved than the four sources studied here (AFGL2591 is a mid-IR bright HMPO, while the 4 others are mid-IR quiet HMPOs), all these are still HMPOs, i.e. not hot molecular cores or ultra-compact HII regions. As far as we know, it is the only large chemical study of such sources so we will use it as a reference. The two figures have been obtained for a different amount of the atomic sulfur initially available on the grain surfaces (models 1 and 2, see 2.3). IRAS 18264, IRAS 18162 and IRAS 05358 show very similar molecular profiles since their temperature and density gradients are similar (Fig. 1), except for radii larger than $7 \times 10^{3} \mathrm{AU}$ where the depletion of the species is more pronounced in IRAS 18162. W43-mm1 shows different abundance profiles, especially the outer envelope where the molecules are more depleted because of higher densities. In this source, both models 1 and 2 lead to smaller abundances of $\mathrm{SO}, \mathrm{H}_{2} \mathrm{~S}$, and $\mathrm{CS}$ molecules compared to the other sources. All species show strong variations in their abundances with radius.

The radius at which the gas and dust temperatures are around 40-50 K represents a transition from an "outer envelope", where the S-bearing molecules are depleted on the grain mantles, and
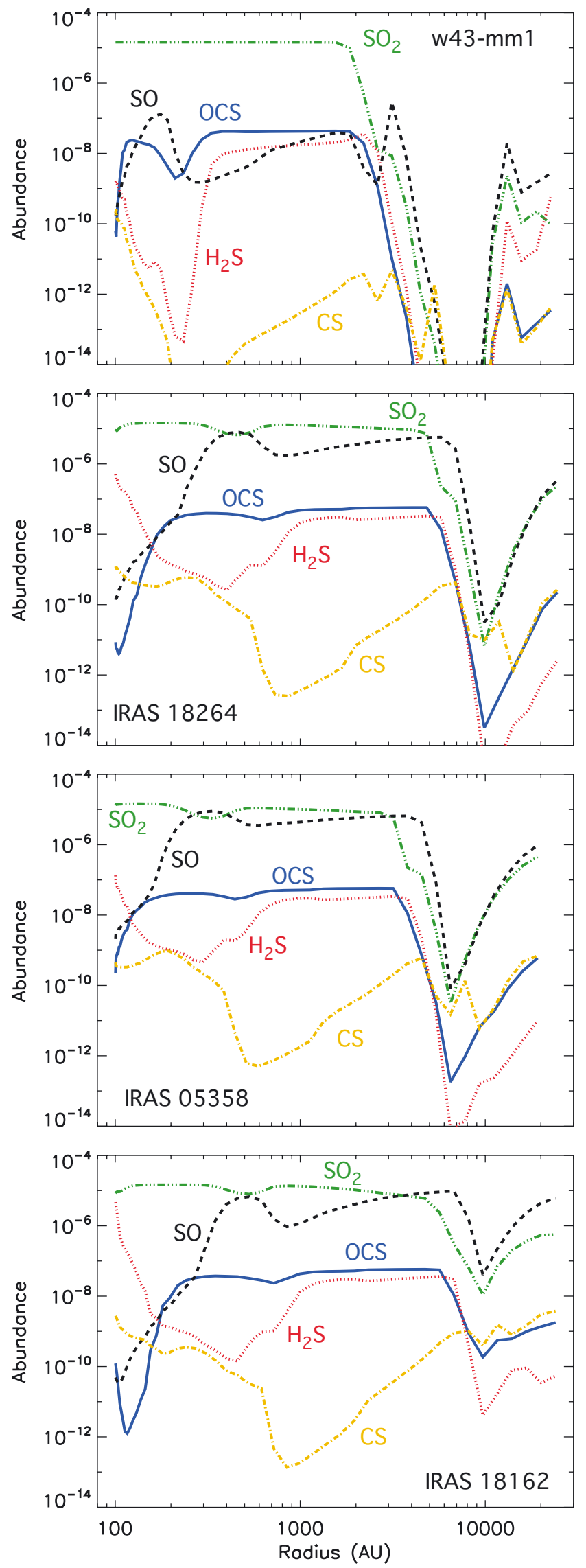

Fig. 2. OCS, $\mathrm{H}_{2} \mathrm{~S}, \mathrm{CS}, \mathrm{SO}$ and $\mathrm{SO}_{2}$ abundance $(/ \mathrm{H})$ profiles as a function of radius from the center in the envelope of four high mass protostars (IRAS 18264, IRAS 18162, IRAS 05358 and W43-MM1). Initial composition of Model 1 is used. Integrated time is $10^{4} \mathrm{yr}$. 
V. Wakelam et al.: Sulfur chemistry: 1D modeling in massive dense cores
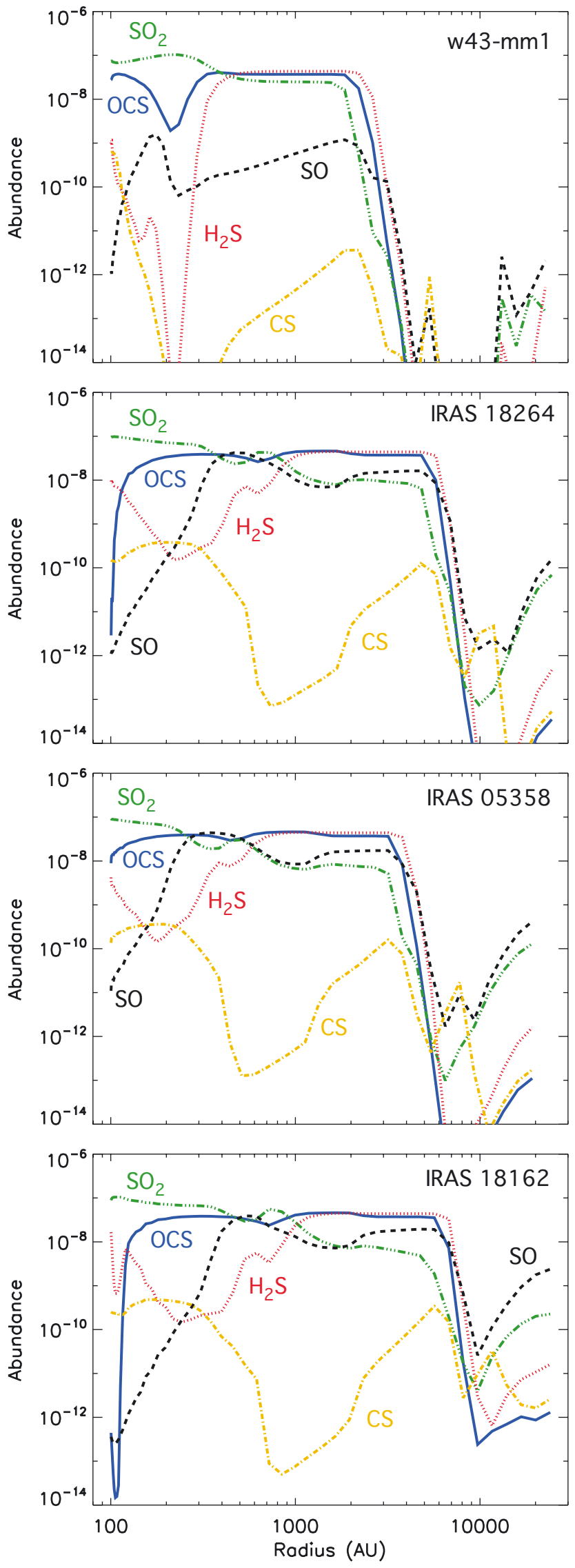

Fig. 3. OCS, $\mathrm{H}_{2} \mathrm{~S}, \mathrm{CS}, \mathrm{SO}$ and $\mathrm{SO}_{2}$ abundance $(/ \mathrm{H})$ profiles as a function of radius from the center in the envelope of four high mass protostars (IRAS 18264, IRAS 18162, IRAS 05358 and W43-MM1). Initial composition of Model 2 is used. Integrated time is $10^{4} \mathrm{yr}$. an "inner envelope" where the abundances of the S-bearing species are much larger. In the inner envelope, towards the center of the protostar, all molecules except $\mathrm{SO}_{2}$ again show a decrease in their abundances at radii depending on the source, the molecule and the model. In IRAS 18264 and model 1 for instance, OCS drops at 200 AU and SO abundance at 400 AU. The $\mathrm{H}_{2} \mathrm{~S}$ abundance decreases by more than one order of magnitude between 1000 and 400 AU before increasing again. In all sources, $\mathrm{SO}_{2}$ is the only species whose abundance is rather constant in the inner envelope, whereas the CS abundance is quite low and shows a strong dip where the temperature is about $200 \mathrm{~K}$ (see discussion in Sect. 4). In the very outer part of the envelopes (at radii larger than $10000 \mathrm{AU}$ ), the species abundances increase again because the density of the gas is lower and species have not had time yet to deplete on the grains.

In model 1, a large quantity of atomic sulfur is evaporated in the gas phase when the temperature is above $\sim 20 \mathrm{~K}$. Atomic sulfur is then quickly converted into $\mathrm{SO}$ by reacting with $\mathrm{O}_{2}$. SO in turn produces $\mathrm{SO}_{2}$ by reactions with $\mathrm{OH}$ and $\mathrm{O}$. For temperatures higher than $\sim 40-50 \mathrm{~K}$, OCS and $\mathrm{H}_{2} \mathrm{~S}$ are evaporated in the gas phase, and their abundances are determined by their initial solid abundances. In model 2 , it is the destruction of $\mathrm{H}_{2} \mathrm{~S}$ through the following paths that releases the necessary sulfur to produce SO and $\mathrm{SO}_{2}$ at high temperature: $\mathrm{H}_{2} \mathrm{~S}+\mathrm{H}_{3} \mathrm{O}^{+} \rightarrow \mathrm{H}_{3} \mathrm{~S}^{+}+\mathrm{H}_{2} \mathrm{O}$, $\mathrm{H}_{3} \mathrm{~S}^{+}+\mathrm{e}^{-} \rightarrow \mathrm{HS}+\mathrm{H}_{2}$ and $\mathrm{HS}+\mathrm{O} \rightarrow \mathrm{SO}+\mathrm{H} . \mathrm{SO}_{2}$ is the dominant $\mathrm{S}$-bearing molecule after a few $10^{4} \mathrm{yr}$ in all models at radii smaller than $7 \times 10^{3} \mathrm{AU}$. We anticipate the comparison with the observation given in Sect. 5 by saying that model 1 is far from reproducing our observations since very low abundances of Sbearing molecules have been observed. For this reason, we only discuss model 2 in the rest of the paper.

Some other species abundances (for model 2 and $10^{4} \mathrm{yr}$ ) are shown in Fig. 5 for the source w43-mm1 and Fig. 6 for the source IRAS 18264 (representative of the IRAS sources of our sample). In IRAS 18264, the CO abundance is constant over the envelope $\left(\sim 8 \times 10^{-5}\right)$ since the temperature is above $20 \mathrm{~K}$, CO depletion occurs whereas at radii larger than $10000 \mathrm{AU}$ in w43-mm1. Inside the protostellar envelopes (radii smaller than $200 \mathrm{AU}$ for w43-mm1 and 600 AU for IRAS 18264), the main reservoir of oxygen is water, except for $\mathrm{CO}$. At larger radii, the oxygen is mainly in the atomic form. This transition between $\mathrm{H}_{2} \mathrm{O}$ and $\mathrm{O}$ corresponds to the dip in the CS abundance (see also Sect. 4). Similarly, the peak in $\mathrm{HOCS}^{+}$, precursor of CS, around $2000 \mathrm{AU}$ in w43-mm1 and $5000 \mathrm{AU}$ in IRAS 18264 is responsible for the peak in the CS abundance.

The abundances in Figs. 2 and 3 are displayed for an age of $10^{4} \mathrm{yr}$. By age, we mean the time during which the chemistry evolves after the temperature and density of the envelope have reached their present state. As an example, the chemical profiles in the source IRAS 18264, for the elemental abundances of model 2, are shown in Fig. 4 for two different chemical ages: $10^{3}$ and $5 \times 10^{4} \mathrm{yr}$. The chemistry evolves quickly inside the protostellar envelopes and the abundances are similar at $10^{3}$ and $10^{4} \mathrm{yr}$ except for the $\mathrm{SO}_{2}$ abundance, which increases significantly with time. As time evolves after $10^{4} \mathrm{yr}$, OCS and CS at radii $2000-6000 \mathrm{AU}$ are destroyed by $\mathrm{HCO}^{+}$. Abundances in the outer parts of the envelope also evolve toward more depletion.

It is interesting to notice that the abundance of OCS does not change much at radii smaller than 2000 AU with time. In fact, it takes more than $10^{5}$ yr to significantly destroy this molecule in the conditions of the studied massive protostars. If OCS were the major carrier of S-bearing molecules on grains as suggested by van der Tak et al. (2003), time scales for the sulfur chemistry would be longer than what we considered here. 

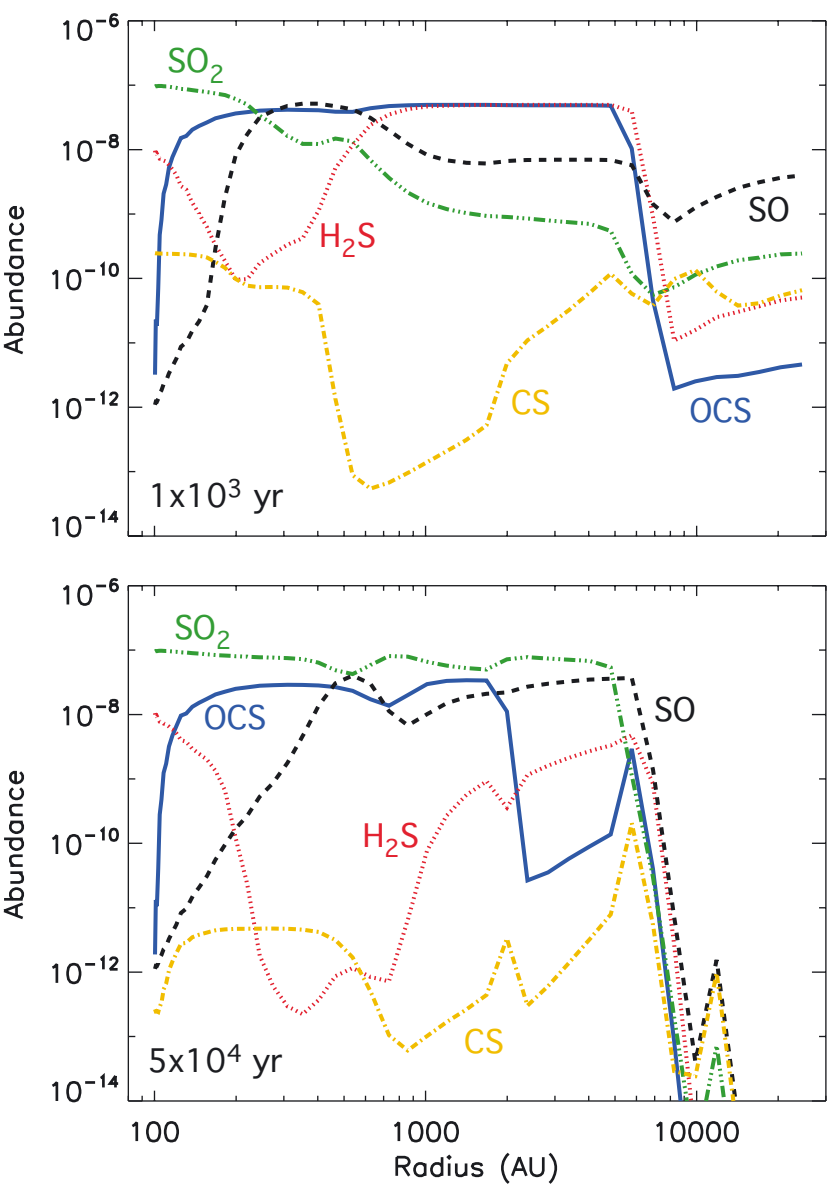

Fig. 4. OCS, $\mathrm{H}_{2} \mathrm{~S}, \mathrm{CS}, \mathrm{SO}$ and $\mathrm{SO}_{2}$ abundance $(/ \mathrm{H})$ profiles as a function of radius from the center in the envelope of IRAS 18264. Initial composition of Model 2 is used. Integrated times are $10^{3} \mathrm{yr}$ for the figure on the top and $5 \times 10^{4} \mathrm{yr}$ for the figure on the bottom.
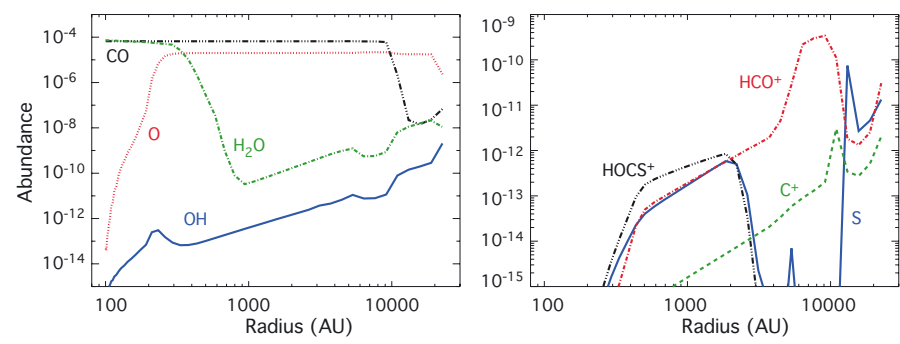

Fig. 5. Abundance $(/ \mathrm{H})$ profiles of a selection of species as a function of radius from the center in the envelope of W43-MM1. Initial composition of Model 2 is used. Integrated time is $10^{4} \mathrm{yr}$.

\subsection{Abundances ratios}

It is common when comparing with observations to use abundance ratios between two observed species rather than abundances compared to $\mathrm{H}_{2}$. We have observational constraints on the abundances at two different radii corresponding to gas temperatures of $60 \mathrm{~K}$ and $100 \mathrm{~K}$ (see Sect. 2.4). The abundance (and abundance ratios) observed at $60 \mathrm{~K}$ are probably not good tracers for the evolution of the protostars since we probe the regions of evaporation versus depletion processes of the sulfurbearing species. To simplify the problem since we have many parameters, we display in Fig. 7 the abundance ratios of SO, OCS, $\mathrm{H}_{2} \mathrm{~S}$, and CS compared to $\mathrm{SO}_{2}$ in IRAS 18264, and W43MM1 as a function of time for the radii corresponding to the
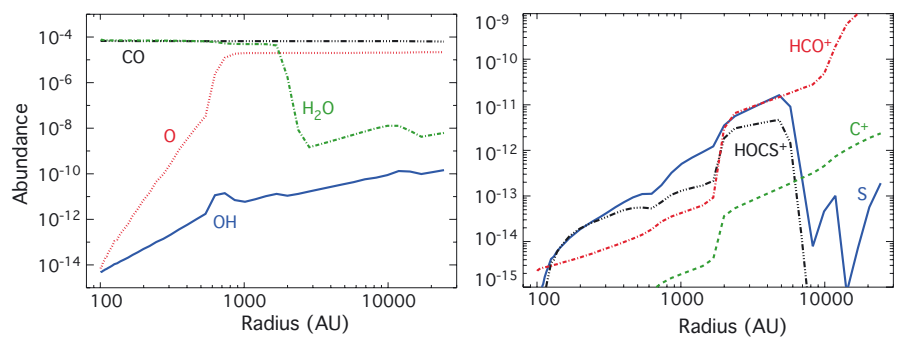

Fig. 6. Abundance $(/ \mathrm{H})$ profiles of a selection of species as a function of radius from the center in the envelope of IRAS 18264. Initial composition of Model 2 is used. Integrated time is $10^{4} \mathrm{yr}$.
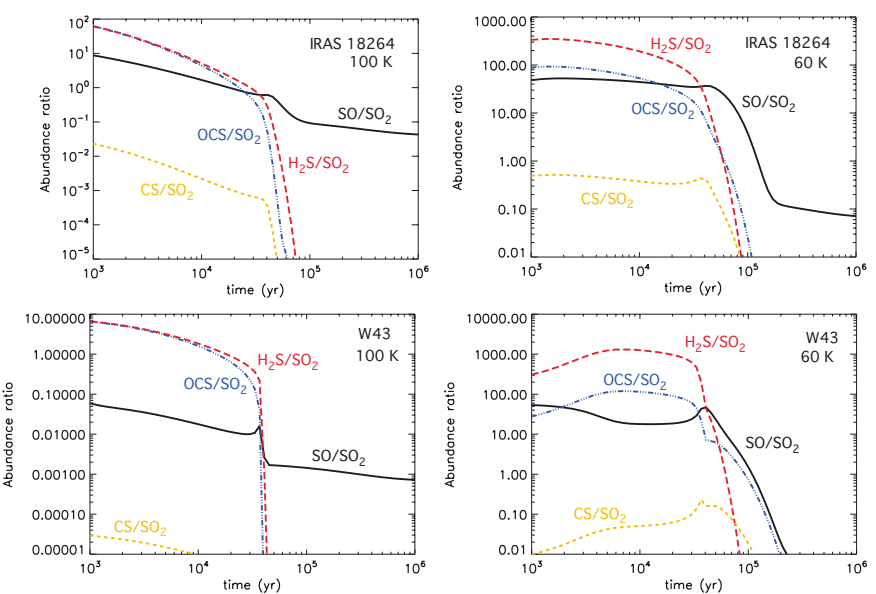

Fig. 7. $\mathrm{SO} / \mathrm{SO}_{2}, \mathrm{OCS} / \mathrm{SO}_{2}, \mathrm{H}_{2} \mathrm{~S} / \mathrm{SO}_{2}$ and $\mathrm{CS} / \mathrm{SO}_{2}$ abundance ratios as a function of time at radius where the temperatures are $60 \mathrm{~K}$ (right panels) and $100 \mathrm{~K}$ (left panels) in IRAS 18264 and W43-MM1. Initial composition of Model 2 is used.

temperature $100 \mathrm{~K}$ (2680 AU in IRAS 18264 and $640 \mathrm{AU}$ in W43-MM1). We did not show the abundance ratios for IRAS 18162 and IRAS 05358 , because they are very similar to those in IRAS 18264. All four abundance ratios decrease with time. $\mathrm{SO} / \mathrm{SO}_{2}$ shows a monotone variation with time contrary to the three other ratios, which drastically decrease at times between $10^{4}$ and $10^{5}$ yr. Since we do not reproduce the observed abundance of $\mathrm{CS}, \mathrm{OCS} / \mathrm{SO}_{2}$ and $\mathrm{H}_{2} \mathrm{~S} / \mathrm{SO}_{2}$ seem to be the best candidates to constrain some evolutionary time for these sources.

\subsection{Influence of diffusion}

The general effect of diffusion is to smooth out the abundance profiles across the envelope and decrease the depletion of the species abundances in the outer parts. Abundance profiles for the source IRAS 18264 and model 2 are shown in Fig. 8 for three different values of the turbulent diffusivity $\kappa: 2 \times 10^{14} \mathrm{~cm}^{-2} \mathrm{~s}^{-1}$, $2 \times 10^{16} \mathrm{~cm}^{-2} \mathrm{~s}^{-1}$, and $2 \times 10^{18} \mathrm{~cm}^{-2} \mathrm{~s}^{-1}$. These figures can be compared to Fig. 3 where $\kappa$ is zero. Our largest $\kappa$ is four orders of magnitude smaller than the maximum value derived in Sect. 2.2, but the effect on the chemistry is already strong. In that case, the abundances of OCS, $\mathrm{H}_{2} \mathrm{~S}, \mathrm{SO}$, and $\mathrm{SO}_{2}$ are then constant and equal up to radius $5 \times 10^{3} \mathrm{AU}$. The $\mathrm{CS}$ abundance is also rather smooth, but four orders of magnitude less than the other species. For smaller $\kappa$, the smoothing is less efficient and becomes negligible for $\kappa$ lower than $2 \times 10^{14} \mathrm{~cm}^{-2} \mathrm{~s}^{-1}$. 

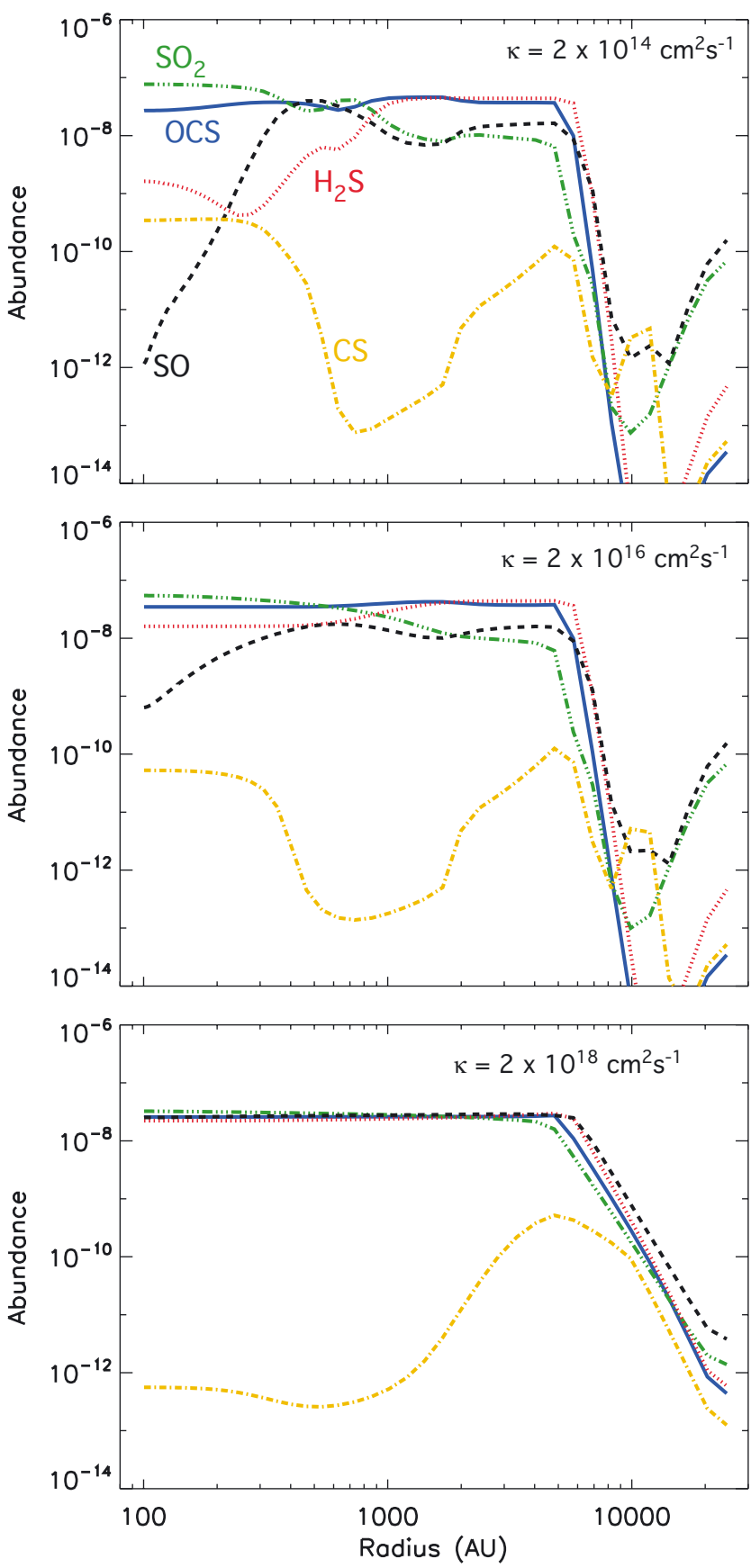

Fig. 8. OCS, $\mathrm{H}_{2} \mathrm{~S}, \mathrm{CS}, \mathrm{SO}$ and $\mathrm{SO}_{2}$ abundance $(/ \mathrm{H})$ profiles as a function of radius from the center in the envelope of IRAS 18264. Initial composition of Model 2 is used. Integrated times is $10^{4} \mathrm{yr}$. Three different turbulent diffusivity $\kappa$ are used for each panel: $2 \times 10^{14} \mathrm{~cm}^{-2} \mathrm{~s}^{-1}$ for the upper panel, $2 \times 10^{16} \mathrm{~cm}^{-2} \mathrm{~s}^{-1}$ for the panel in the middle and $2 \times 10^{18} \mathrm{~cm}^{-2} \mathrm{~s}^{-1}$ for the lower panel.

\section{The CS abundance}

As already noticed, we predict that CS is much less abundant than the other S-bearing species contrary to the observations, which show abundances of the same order for all species (see also Wakelam et al. 2004). In our model, CS is produced at high temperature by the dissociative recombination of $\mathrm{HCS}^{+}$ in the central part where the temperature is above $300 \mathrm{~K}$ and the dissociative recombination of $\mathrm{HOCS}^{+}$at lower temperature. $\mathrm{HCS}^{+}$is itself produced by $\mathrm{C}^{+}+\mathrm{H}_{2} \mathrm{~S}$ and $\mathrm{HOCS}^{+}$by $\mathrm{HCO}^{+}+$ OCS and $\mathrm{H}_{3}^{+}+$OCS. For the destruction, we also have two regimes: $\mathrm{CS}+\mathrm{H}^{+}$at temperatures higher than $300 \mathrm{~K}$ and $\mathrm{CS}$ + O for lower temperatures. Charnley (1997) and Hatchell et al. (1998) have produced much more CS than in this work because they did not include any atomic oxygen in their initial conditions. Except for helium and hydrogen, they considered that all species were already in a molecular form. Oxygen was then in the form of $\mathrm{CO}, \mathrm{H}_{2} \mathrm{O}$, and $\mathrm{O}_{2}$. Contrary to what was said in Wakelam et al. (2004), changing the rate coefficient of the dissociation of $\mathrm{CO}$ by secondary photons produced by cosmic-rays does not change the abundance of CS predicted at high temperature. Since this problem is not seen in cold dense sources (see for instance Wakelam et al. 2006), we may be missing some formation paths for CS at high temperature. Some rate coefficients may also not be accurate for the temperature range where we are using them. As an example, some new recommendations for the rate coefficient of the neutral-neutral reaction $\mathrm{O}+$ CS for temperatures between 150 and $300 \mathrm{~K}$ have been posted on the KIDA database (http: //kida.obs.u-bordeaux1.fr) following this work. However, the small proposed changes in this rate coefficient does not change the gas-phase CS abundance in our simulations.

That we do not reproduce the CS abundance at $100 \mathrm{~K}$ may also come from our other assumptions in our modeling such as our initial conditions, the temperature and density profiles, the brutal rise in the temperature and density from the molecular cloud stage. This, however, should not influence the abundance of the other S-bearing molecules by more than a factor of a few since they all have similar observed abundances. The CS problem may also be related to the form of sulfur on grains. Recent laboratory experiments show that $\mathrm{H}_{2} \mathrm{~S}$ on ISM analog surfaces are easily destroyed by energetic particles, and surface $\mathrm{S}$ then recombines to form OCS, $\mathrm{SO}_{2}, \mathrm{C}_{2} \mathrm{~S}$, and a majority of sulfur-rich residuum (Garozzo et al. 2010). The surface $\mathrm{C}_{2} \mathrm{~S}$ may then be evaporated in the gas phase and contribute to the CS chemistry.

Last but not least, we stress that the discrepancy might also originate in the observed abundances. The observed abundance of CS representative of the $100 \mathrm{~K}$ layers in the protostellar envelopes was derived using the CS (7-6) line emission, which has an upper level energy of $65.8 \mathrm{~K}$. First of all, these abundances are derived independently for each observed transition of each species, assuming that the emission for a given line mostly comes from one single place at a given temperature and density, whereas lines are excited over several layers. Considering the upper level energy of CS (7-6), it is likely that this transition is also excited in the layers of the protostellar envelopes at temperatures below $100 \mathrm{~K}$ where our chemical model predicts larger abundances of CS. The CS abundance is predicted by our chemical models to vary much more with the radius than for the other S-bearing abundances at these temperatures. As a result, an uncertainty about the location of the emission may lead to a larger uncertainty concerning the abundance. Furthermore, this line was blended with an $\mathrm{H}_{2} \mathrm{CO}$ line, and the procedure to separate lines did not work well in IRAS 18264 and w43-mm1 (see Herpin et al. 2009). The CS abundances at $100 \mathrm{~K}$ derived by (Herpin et al. 2009) may then be overestimated, and one would need to observe higher energy transitions of CS (unfortunately not accessible from the ground) to confirm its abundance at high temperature.

\section{Comparison with observations}

In this section, we discuss the comparison between our modeling and observations of S-bearing molecules in the four high-mass protostars from Herpin et al. (2009). As a summary, very small 
abundances have been found compared to the cosmic abundance of sulfur $\left(1.5 \times 10^{-5}\right.$ compared to $\left.\mathrm{H}\right)$. The sum of all observed $\mathrm{S}$ bearing species is only $2 \times 10^{-9}$ to $5 \times 10^{-8}$. This result suggests that we are closer to model 2 than model 1, i.e. a large part of the sulfur in this object is in a stable, not observed form and does not participate in the gas phase chemistry. We discuss this point in the next section. We are far from reproducing all the observed abundances correctly. We overproduce them by more than one order of magnitude even using model 2, except for CS which is strongly underestimated.

The OCS/SO $\mathrm{S}_{2}, \mathrm{H}_{2} \mathrm{~S} / \mathrm{SO}_{2}$, and $\mathrm{SO} / \mathrm{SO}_{2}$ abundance ratios could in theory be used to trace the age of these sources. We report in Fig. 9 the abundance ratios predicted by model 2 as a function of time for the radius corresponding to $100 \mathrm{~K}$. As stated in Sect. 3.2, the abundances derived at radii where the temperature is about $60 \mathrm{~K}$ trace regions of the envelopes where the depletion processes compete with the evaporation from the grains. For this reason we focus on the abundances obtained for shells of gas at higher temperature probably more representative of the evolution of the protostar itself. In the same figures we plot the ratios observed in each source. The OCS/SO 2 and $\mathrm{H}_{2} \mathrm{~S} / \mathrm{SO}_{2}$ abundance ratios are above the observed ones until a few $10^{4} \mathrm{yr}$ and then decrease sharply to the observational values and below. As a consequence, in the observed range for these ratios, the constraint on the age is very strong. This time depends on the physical properties of the source. The three IRAS sources being very similar, the time of decrease is almost the same. W43MM1 is denser and the time at which the abundance ratios drop is less. The constraint on the relative age of the sources provided by the observed ratios OCS/SO $\mathrm{S}_{2}$ and $\mathrm{H}_{2} \mathrm{~S} / \mathrm{SO}_{2}$ is in good agreement with the evolution sequence W43-MM1 $\rightarrow$ IRAS 18264 $\rightarrow$ IRAS $05358 \rightarrow$ IRAS 18162 proposed using other methods (see Herpin et al. 2009). If one considers the uncertainties in both the observed (Herpin et al. 2009) and modeled (Wakelam et al. 2005) abundance ratios, no definitive conclusion can be drawn from these observations. The constraints on W43-MM1 are maybe more robust and indicate that the source is younger than the three other ones. One important conclusion here is that more than the age of the source, it is the physical conditions in the envelope that directly determine the variation in the OCS, $\mathrm{H}_{2} \mathrm{~S}$, and $\mathrm{SO}_{2}$ abundance. There is of course a relation between the age and the physical conditions. OCS/SO $\mathrm{SO}_{2}$ and $\mathrm{H}_{2} \mathrm{~S} / \mathrm{SO}_{2}$ can, however, not be used to compare protostars that are close in age. The $\mathrm{SO} / \mathrm{SO}_{2}$ abundance ratios are more sensitive to time. We do need a very young chemical model for W43-MM1 to reproduce the observed abundance ratio in this source, which does not agree with the other constraints. The $\mathrm{SO} / \mathrm{SO}_{2}$ abundance ratios observed in IRAS 18162 and IRAS 18264 are also too close to conclude anything about their age.

Observations also seem to indicate significant variations in the species abundances with the radius in a source. $\mathrm{H}_{2} \mathrm{~S}$, for instance, was observed to vary by one order of magnitude in IRAS 18264 for radii between $2.6 \times 10^{3}$ and $5.4 \times 10^{3} \mathrm{AU}$, which is approximately reproduced by our model. Including diffusion smooths the predicted abundance profiles. If chemical variations are so strong across these objects, then we would expect the turbulent diffusivity to be smaller than $2 \times 10^{14} \mathrm{~cm}^{2} \mathrm{~s}^{-1}$. Supersonic velocities due to diffusion have been observed in these sources. Assuming a turbulent velocity of about $1 \mathrm{~km} \mathrm{~s}^{-1}$ as observed, the maximum mixing length would be a few $10^{-4} \mathrm{AU}$, much smaller than what we would expect. The very small mixing efficiency associated with the observed nonthermal velocity raises questions about the nature of this "turbulence". Either turbulence has a very peculiar nature, or more likely, the observed structure is
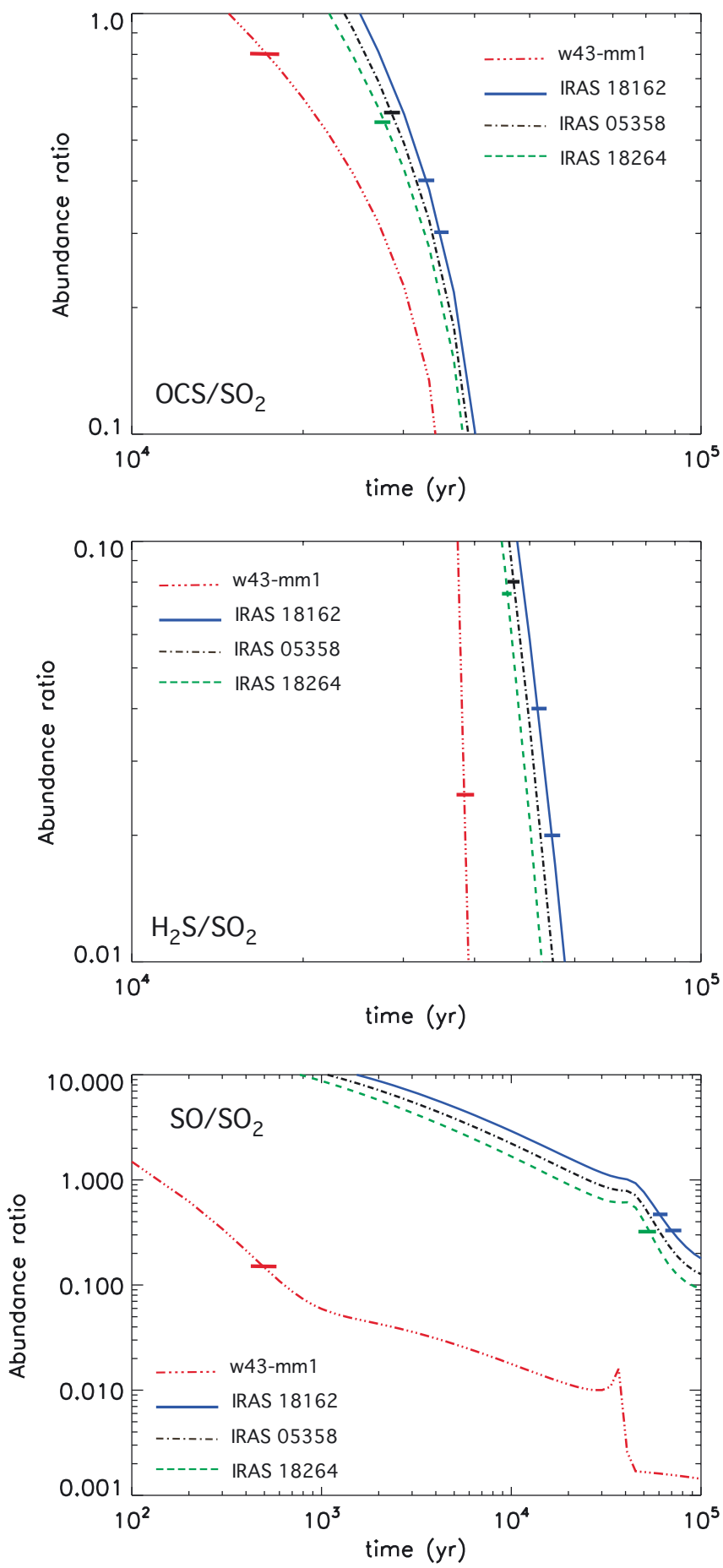

Fig. 9. OCS/ $\mathrm{SO}_{2}$ (upper panel), $\mathrm{H}_{2} \mathrm{~S} / \mathrm{SO}_{2}$ (middle panel) and $\mathrm{SO} / \mathrm{SO}_{2}$ (lower panel) abundance ratios as a function of time at radius where the temperature is $100 \mathrm{~K}$ in the four studied sources. Initial composition of Model 2 is used. For each source, the observed abundance ratio is reported on the curves as horizontal thick line. Two values are drawn for IRAS 18162 as two $\mathrm{SO}_{2}$ abundances have been determined using two different lines (see Herpin et al. 2009). No observed value $\mathrm{SO} / \mathrm{SO}_{2}$ is reported for IRAS 05358.

unresolved and composed of smaller structures of varying turbulence intensity. Of course, nonthermal motions can be produced by a variety of processes (rotation, infall, outflow, etc.), which will not always induce an efficient mixing, but can still be mistaken for turbulence, at least partly. 


\section{Conclusions}

We performed a 1D chemical modeling of the sulfur-bearing species in four young high-mass protostars (IRAS 18264, IRAS 18162, IRAS 05358, and W43-MM1) using temperature and density profiles determined from their SED by Herpin et al. (2009). In addition to the time-dependent chemistry, we studied the effect of a turbulent-like mixing on the abundance profiles through the envelopes. Here are our main conclusions:

- Sulfur chemistry depends strongly on the 1D physical conditions. Any observed set of abundances should be compared with a chemical model computed with the same temperature and density traced by the observations.

- To use sulfur chemistry as chemical clocks, observations tracing the gas at temperatures higher than $70 \mathrm{~K}$ should be used to avoid confusion with depletion mechanisms. At lower temperatures, S-bearing molecules stick on grains so that their abundance in the gas phase will also depend upon this process.

- In our case, no conclusion can be drawn on the relative age of IRAS 18264, IRAS 18162 and IRAS 05358 if one considers the uncertainties in the observed and modeled abundances because these sources are too close in age. W43MM1 seems, in contrast, younger than the other sources.

- Turbulent mixing could occur in young high-mass protostars on a too small geometric scale to affect the chemical abundance profiles through the envelope. Such a small scale suggests that either turbulence is very unusual or the structure is unresolved. There is a possibility that at least some of the nonthermal broadening of lines would be the consequence of a non turbulent process, inducing a very inefficient mixing inside the protostellar envelopes.

- The CS molecule is predicted by our chemical models to be less abundant than the other S-bearing species by several orders of magnitude at temperatures above $100 \mathrm{~K}$ (see also Wakelam et al. 2004). This seems to disagree with the observations, although the observed abundances from Herpin et al. (2009) may be overestimated. If the disagreement were true, however, this suggests that some formation paths are missing in our networks at high temperature.

- Our comparisons between observations and chemical modeling seem to indicate that the majority of the sulfur is still in a refractory form on grains. It is interesting to note that Wakelam et al. (2004) also need a depletion by a factor of ten of the elemental sulfur abundance to reproduce observations in Orion-KL, whereas no depletion is mandatory to reproduce the observations in the low-mass protostar IRAS 16293-2422. Based on laboratory experiments, Garozzo et al. (2010) have recently suggested that $\mathrm{H}_{2} \mathrm{~S}$ on interstellar grains are easily destroyed by energetic particles to form $\mathrm{OCS}, \mathrm{SO}_{2}$, and a majority of sulfur-rich residuum, which could be polymers of sulfur or amorphous aggregates of sulfur, as suggested by Wakelam et al. (2004). In the environments of high-mass star formation, interstellar ices are probably exposed to stronger particle fluxes so that a larger quantity of the atomic sulfur could be converted in refractory form. The identification of the sulfur-rich residuum found by Garozzo et al. (2010) could certainly bring new insight into the reservoir of sulfur in the interstellar medium.

Acknowledgements. We thank the referee for his careful reading of the paper and his suggestions. V.W. acknowledges the French CNRS program PCMI for partial support of this work. Some kinetic data we used have been downloaded from the online database KIDA (KInetic Database for Astrochemistry, http:// kida.obs.u-bordeaux1.fr).

\section{References}

Boogert, A. C. A., Helmich, F. P., van Dishoeck, E. F., et al. 1998, A\&A, 336, 352

Buckle, J. V., \& Fuller, G. A. 2003, A\&A, 399, 567

Charnley, S. B. 1997, ApJ, 481, 396

Chiar, J. E., Adamson, A. J., \& Whittet, D. C. B. 1996, ApJ, 472, 665

Collings, M. P., Anderson, M. A., Chen, R., et al. 2004, MNRAS, 354, 1133

Doty, S. D., \& Neufeld, D. A. 1997, ApJ, 489, 122

Doty, S. D., van Dishoeck, E. F., van der Tak, F. F. S., \& Boonman, A. M. S. 2002, A\&A, 389, 446

Garozzo, M., Fulvio, D., Kanuchova, Z., Palumbo, M. E., \& Strazzulla, G. 2010, A\&A, 509, A67

Gibb, E. L., Whittet, D. C. B., Schutte, W. A., et al. 2000, ApJ, 536, 347

Hartquist, T. W., Dalgarno, A., \& Oppenheimer, M. 1980, ApJ, 236, 182

Hasegawa, T. I., \& Herbst, E. 1993, MNRAS, 261, 83

Hasegawa, T. I., Herbst, E., \& Leung, C. M. 1992, ApJS, 82, 167

Hatchell, J., Thompson, M. A., Millar, T. J., \& MacDonald, G. H. 1998, A\&A, 338,713

Herpin, F., Marseille, M., Wakelam, V., Bontemps, S., \& Lis, D. C. 2009, A\&A, 504,853

Hollenbach, D., \& McKee, C. F. 1979, ApJS, 41, 555

Jenkins, E. B. 2009, ApJ, 700, 1299

Keane, J. V., Tielens, A. G. G. M., Boogert, A. C. A., Schutte, W. A., \& Whittet, D. C. B. 2001, A\&A, 376, 254

Leung, C. M., Herbst, E., \& Huebner, W. F. 1984, ApJS, 56, 231

Marseille, M., Bontemps, S., Herpin, F., van der Tak, F. F. S., \& Purcell, C. R. 2008, A\&A, 488, 579

Masunaga, H., \& Inutsuka, S. 2000, ApJ, 531, 350

Motte, F., Bontemps, S., Schilke, P., et al. 2007, A\&A, 476, 1243

Pagani, L., Olofsson, A. O. H., Bergman, P., et al. 2003, A\&A, 402, L77

Palumbo, M. E., Geballe, T. R., \& Tielens, A. G. G. M. 1997, ApJ, 479, 839

Pineau Des Forêts, G., Roueff, E., Schilke, P., \& Flower, D. R. 1993, MNRAS, 262, 915

Prandtl, L. 1925, Zs. angew. Math. Mech., 5, 136

Ruffle, D. P., Hartquist, T. W., Caselli, P., \& Williams, D. A. 1999, MNRAS, 306, 691

Ruffle, D. P., Rae, J. G. L., Pilling, M. J., Hartquist, T. W., \& Herbst, E. 2002, A\&A, 381, L13

Scappini, F., Cecchi-Pestellini, C., Smith, H., Klemperer, W., \& Dalgarno, A. 2003, MNRAS, 341, 657

Schutte, W. A., Tielens, A. G. G. M., Whittet, D. C. B., et al. 1996, A\&A, 315, L333

Snell, R. L., Howe, J. E., Ashby, M. L. N., et al. 2000, ApJ, 539, L101

Sofia, U. J., \& Meyer, D. M. 2001, ApJ, 554, L221

van der Tak, F. F. S., \& van Dishoeck, E. F. 2000, A\&A, 358, L79

van der Tak, F. F. S., Boonman, A. M. S., Braakman, R., \& van Dishoeck, E. F. 2003, A\&A, 412, 133

van Dishoeck, E. F., \& Blake, G. A. 1998, ARA\&A, 36, 317

Wakelam, V., \& Herbst, E. 2008, ApJ, 680, 371

Wakelam, V., Caselli, P., Ceccarelli, C., Herbst, E., \& Castets, A. 2004, A\&A, 422, 159

Wakelam, V., Selsis, F., Herbst, E., \& Caselli, P. 2005, A\&A, 444, 883

Wakelam, V., Herbst, E., \& Selsis, F. 2006, A\&A, 451, 551

Wakelam, V., Herbst, E., Le Bourlot, J., et al. 2010, A\&A, 517, A21

Wolf, S., Henning, T., \& Stecklum, B. 1999, A\&A, 349, 839 